\title{
BIEBERBACH THEOREMS FOR SOLVABLE LIE GROUPS*
}

\author{
KAREL DEKIMPE ${ }^{\dagger}$, KYUNG BAI LEE ${ }^{\ddagger}$, AND FRANK RAYMOND $§$
}

1. Introduction. Let $G$ be a Lie group and let $\operatorname{Aut}(G)$ be the group of continuous automorphisms of $G$. The group $\operatorname{Aff}(G)$ is the semi-direct product $\operatorname{Aff}(G)=$ $G \rtimes \operatorname{Aut}(G)$ with multiplication

$$
(a, \alpha) \cdot(b, \beta)=(a \cdot \alpha(b), \alpha \beta) .
$$

It has a Lie group structure and acts on $G$ by

$$
(a, \alpha) \cdot x=a \cdot \alpha(x)
$$

for all $x \in G$. With the linear connection on $G$ defined by the left invariant vector fields, it is known that $\operatorname{Aff}(G)$ is the group of connection-preserving diffeomorphisms of $G$, see [KT] Proposition 2.1.

TERMINOLOGY. In this paper we will use the term lattice of a Lie group $G$, to denote a discrete cocompact subgroup of $G$. Further, we will say that an automorphism $\alpha$ of a Lie group $G$ is unipotent if and only if its differential do (in the automorphism group of the corresponding Lie algebra $\mathfrak{G}$ ) is unipotent. Analogously we will speak of an element acting unipotently on a Lie group $G$.

For $G=\mathbb{R}^{n}$, the following three theorems have been proven by Bieberbach. See $[\mathrm{W}]$ or $[\mathrm{C}]$ :

THEOREM $1^{\prime}$. Let $\pi \subset \mathbb{R}^{n} \rtimes \mathrm{O}(n)$ be a lattice. Then $\Gamma=\pi \cap \mathbb{R}^{n}$ is a lattice of $\mathbb{R}^{n}$, and $\Gamma$ has finite index in $\pi$.

THEOREM $2^{\prime}$. Let $\pi, \pi^{\prime} \subset \mathbb{R}^{n} \rtimes \mathrm{O}(n)$ be lattices. Then every isomorphism $\theta$ : $\pi \rightarrow \pi^{\prime}$ is a conjugation by an element of $\mathbb{R}^{n} \rtimes \mathrm{GL}(n, \mathbb{R})$.

THEOREM $3^{\prime}$. Under each torus $\mathbb{Z}^{n} \backslash \mathbb{R}^{n}$, there are only finitely many flat manifolds which are covered by the torus.

All three Bieberbach theorems have been generalized to the situation where $G$ is a simply connected, connected nilpotent Lie group ([A1], [LR2], [DIM], [D2]). It is therefore natural to investigate the situation for solvable $G$. Unfortunately, as an example in this paper will show, the first Bieberbach theorem causes problems, even for the class of solvable Lie groups of type $(\mathrm{R})$, a class of groups sharing many properties with the class of nilpotent Lie groups. A connected solvable Lie group $G$ is called of type $(\mathrm{R})$ (or completely solvable) if $\operatorname{ad}(X): \mathfrak{G} \rightarrow \mathfrak{G}$ has only real eigenvalues for each $X \in \mathfrak{G}$. A connected solvable Lie group $G$ is called of type (E) (or exponential) if $\exp : \mathfrak{G} \rightarrow G$ is surjective. Some important properties of such groups are listed below.

Although we will show that there are problems in generalizing the first Bieberbach Theorem, we are able to show which Lie groups of type (E) allow a straightforward generalization of the first Bieberbach theorem (Theorem 1'). In this respect, we need the following definition.

* Received September 4, 2000; accepted for publication October 2, 2000.

† Katholieke Universiteit Leuven, Campus Kortrijk, B-8500 Kortrijk, Belgium (Karel.Dekimpe@ kulak.ac.be). The first author is a Postdoctoral Fellow of the Fund for Scientific Research-Flanders (Belgium) (F.W.O.).

$\ddagger$ University of Oklahoma, Norman, OK 73019, U.S.A. (kblee@math.ou.edu).

$\S$ University of Michigan, Ann Arbor, MI 48109, U.S.A. (fraymond@math.lsa.umich.edu). 
DEFINITION 1. Let $G$ be a connected, simply connected solvable Lie group with nilradical $N$. We say that $G$ has the strong lattice property if for any torus $T \subseteq \operatorname{Aut}(G)$ and for any lattice $\Gamma$ of $G \rtimes T$, there exists a lattice $\tilde{\Gamma}$ of $G$ such that $N \cap \Gamma=N \cap \tilde{\Gamma}$.

The main result of our paper can now be formulated as follows:

THEOREM A. Let $G$ be a connected, simply connected solvable Lie group of type (E) and let $C$ be a compact subgroup of $\operatorname{Aut}(G)$. If $G$ has the strong lattice property and if $\pi \subset G \rtimes C$ is a lattice, then $\Gamma=\pi \cap G$ is a lattice of $G$, and $\Gamma$ has finite index in $\pi$.

REMARK 1. Obviously, Theorem A states that the first Bieberbach theorem generalizes to a given Lie group $G$ of type (E) if and only if $G$ has the strong lattice property.

To justify our interest in solvable groups of type (R) and (E), we now recall some properties of the groups. See [G1], [G2] and [OV] for more details.

(1) Abelian $\Longrightarrow$ Nilpotent $\Longrightarrow$ type $(\mathrm{R}) \Longrightarrow$ type (E).

(2) (Unique Lattice Isomorphism Extension Property) Let $G$ and $G^{\prime}$ be of type (R), and let $\Gamma$ and $\Gamma^{\prime}$ be lattices of $G$ and $G^{\prime}$, respectively. Then any isomorphism from $\Gamma$ to $\Gamma^{\prime}$ extends uniquely to an isomorphism of $G$ onto $G^{\prime}$.

(3) If $G$ is of type (E), then

(a) for arbitrary $X \in \mathfrak{G}, \operatorname{ad}(X): \mathfrak{G} \rightarrow \mathfrak{G}$ has no pure imaginary eigenvalues.

(b) for any $g \in G$ the operator $\operatorname{Ad}(g)$ has no eigenvalues of modulus 1 but different from 1 .

(c) the center of $G$ is connected.

(d) the maximal compact subgroup of $G$ is the unique maximal torus contained in the center of $G$.

Theorem $2^{\prime}$ has been generalized to solvable Lie groups of type (R).

THEOREM 2 [L; Theorem 3.1]. Let $G$ be a connected, simply connected solvable Lie group of type (R), and $C$ be a compact subgroup of $\operatorname{Aut}(G)$. Let $\pi, \pi^{\prime} \subset G \rtimes C$ be lattices, which are finite extensions of lattices of $G$. Then every isomorphism $\theta: \pi \rightarrow$ $\pi^{\prime}$ is a conjugation by an element of $G \rtimes \operatorname{Aut}(G)$.

Note that in the above theorem, we need to assume that a generalization of Theorem $1^{\prime}$ is valid.

In order to generalize Theorem $3^{\prime}$ to a more general class of manifolds, we need a concept of "essential covering" for obvious reasons. A covering $M \rightarrow M^{\prime}$ is called essential if no element of the deck transformation group is homotopic to the identity.

THEOREM 3 [L; Theorem 5.2]. Let $G$ be a connected, simply connected solvable Lie group of type $(\mathrm{R})$, and $\Gamma$ be a lattice of $G$. Then there are only finitely many infra-solvmanifolds which are essentially covered by the solvmanifold $\Gamma \backslash G$.

As already explained, the purpose of this paper is to study the possibility of extending Theorem $1^{\prime}$ to solvable Lie groups. (We do not restrict our attention to groups of type (R), but we also consider groups of type (E)). In other words, we investigate the validity of the following statement:

STATEMENT 1. Let $G$ be a connected, simply connected solvable Lie group and let $C$ be a compact subgroup of $\operatorname{Aut}(G)$. If $\pi \subset G \rtimes C$ is a lattice, then $\Gamma=\pi \cap G$ is a lattice of $G$, and $\Gamma$ has finite index in $\pi$.

2. Generalizing the first Bieberbach theorem. In this section we will prove Theorem A. Before we can actually start the proof of this theorem, we need to establish 
three technical lemmas which will be used later. The third lemma will use the setup of Auslander [A2]; Theorem 2.

LEMMA 1. Let $G$ be a connected, simply connected solvable Lie group of type (E) with nilradical $N$. Then $N$ is maximal nilpotent in $G$. Consequently, the elements $g \in G$ acting unipotently on $N$ (via conjugation in $G$ ) are precisely all the elements of $N$.

Proof. Let $h$ be an element of $G$. The group generated by $N$ and $h$ is nilpotent if and only if $h$ acts unipotently on $N$. As $G / N$ is abelian this is equivalent to $\operatorname{Ad}(h)$ being unipotent (i.e. only has 1 as an eigenvalue). $\operatorname{As} \operatorname{Ad}(h)=\exp \operatorname{ad}_{\log (h)}$ (note that the exponential mapping is a bijection in our case, so log is well defined), this is in turn equivalent to $\operatorname{ad}_{\log (h)}$ being nilpotent. From this it now follows that for any element $h^{\prime}$ in the one parameter subgroup $H$ passing through $h, \operatorname{ad}_{\log \left(h^{\prime}\right)}$ is nilpotent. This implies that the group $N H$ is a connected, nilpotent normal subgroup of $G$, which must be a part of $N$ and so $h \in N$.

LEMMA 2. Let $T$ be a torus and suppose that $A_{1}$ is a lattice of $\mathbb{R}^{n}$ and $A_{2}$ is a finitely generated subgroup of $\mathbb{R}^{n} \oplus T$.

Let $f: \mathbb{R}^{n} \oplus T \rightarrow \mathrm{GL}(k, \mathbb{R})$ be a faithful continuous representation, such that $A_{1}$ and $A_{2}$ leave the same uniform lattice of $\mathbb{R}^{k}$ invariant, then $A_{2} \cap \mathbb{R}^{n}$ is of finite index in $A_{2}$.

Proof. Without loss of generality, we can assume that $A_{1}$ and $A_{2}$ leave the standard lattice $\mathbb{Z}^{k}$ invariant (i.e. $f\left(A_{i}\right) \subset \mathrm{GL}(k, \mathbb{Z})$, for $i=1,2$ ).

We consider the usual metric $d$ on $\mathrm{GL}(k, \mathbb{R})$ obtained by regarding $\mathrm{GL}(k, \mathbb{R})$ as being a subspace of $\mathbb{R}^{k^{2}}$, with the usual Euclidean metric.

Pick any element $a_{2} \in A_{2}$, and consider the group $A$ generated by $A_{1}$ and $a_{2}$. Then $f(A) \subset \mathrm{GL}(k, \mathbb{Z})$. Since $\mathrm{GL}(k, \mathbb{Z})$ is a lattice of $\mathrm{GL}(k, \mathbb{R}), f(A)$ is discrete. However, $f$ is continuous and faithful so that the preimage of a discrete subset is discrete. Therefore, $A$ is discrete in $\mathbb{R}^{n} \oplus T$. Since $A_{1}$ is cocompact in $\mathbb{R}^{n} \oplus T$ already, $A_{1}$ has finite index in $A$. In particular, the $T$-component of $a_{2} \in \mathbb{R}^{n} \oplus T$ has finite order. Since $A_{2}$ is finitely generated, this shows that $A_{2} \cap \mathbb{R}^{n}$ is of finite index in $A_{2}$.

Lemma 3. Let $G$ be a connected, simply connected solvable Lie group of type (E) with nilradical $N$ and let $C$ be a compact subgroup of $\operatorname{Aut}(G)$. Let $\pi$ be a lattice of $G \rtimes C$. Denote by $G^{*}$ the identity component of the closure of $\pi G$ in $G \rtimes C$ and let $\pi^{*}=\pi \cap G^{*}$. then

(1) $\pi^{*}$ has finite index in $\pi$,

(2) $G^{*}$ is solvable,

(3) the nilradical $N^{*}$ of $G^{*}$ coincides with $N$ and

(4) $\pi^{*} \cap N=\pi \cap N$ is a lattice of $N$.

Proof. The first claim is obvious. Theorem 1 of [A2] states that $G^{*}$ is solvable, explaining our second statement. Since $G^{*} / G$ is a compact solvable Lie group, $G^{*} / G=$ $T$ is abelian, thus $G^{*}=G \rtimes T$ and the commutator subgroup of $G^{*},\left[G^{*}, G^{*}\right]$ is contained in $G$. Since $N$ is a characteristic subgroup of $G$, it is normal in $G^{*}$ and hence $N \subset N^{*}$. Moreover, as $N$ is maximal nilpotent in $G$ (Lemma 1), $G \cap N^{*}=N$. It follows that the canonical map $N^{*} / N \rightarrow G^{*} / G=T$ is faithful. Therefore, $N^{*} / N$ is compact, and so we can write $N^{*}=N \cdot T^{*}$ for some torus $T^{*} \subset G^{*}$. (We note here that in the proof of Theorem 2 in [A2], Auslander seems to claim that $N^{*} / N$ is compact, also in case $G$ is not of type (E). However, as Example 1 will show this need not be true.) By (1) and (3)(d) of the properties of a completely solvable Lie group, $T^{*}$ is in the center of $N^{*}$ so that the action of $T^{*}$ on $N$ is trivial, and $N^{*}$ splits as a 
direct product $N^{*}=N \times T^{*}$.

We claim that $T^{*}$ is trivial. For any $t \in T^{*}, x \in N$ and $y \in G$,

$$
\begin{aligned}
{[t, y] \cdot x \cdot[t, y]^{-1} } & =t y t^{-1}\left(y^{-1} \cdot x \cdot y\right) t y^{-1} t^{-1} \\
& =t y\left(y^{-1} \cdot x \cdot y\right) y^{-1} t^{-1} \\
& \left(\text { since } y^{-1} \cdot x \cdot y \in N \text { and } t \text { acts trivially on } N\right) \\
& =t \cdot x \cdot t^{-1} \\
& =x .
\end{aligned}
$$

This shows that $[t, y]$ lies in the centralizer of $N$. However, $[t, y] \in\left[N^{*}, G\right] \subset N^{*} \cap G=$ $N$. Consequently, $[t, y]$ lies in the center of $N$. We have

$$
\left[t^{p}, y\right]=[t, y]^{p} \in N
$$

for all $p \in \mathbb{N}$. Thus, for any $t \in T^{*}$ of finite order, we must have $[t, y]=1$. However, the set of torsion elements is dense in $T^{*}$. Therefore, $[t, G]=1$ for all $t \in T^{*}$, i.e. $T^{*}$ acts trivially on $G$. As $T^{*}$ is a compact subgroup, it is conjugated inside $G^{*}$ to a subgroup of (the maximal compact group) $T$. Let $g_{*} \in G^{*}$ be such that $g_{*} T^{*} g_{*}^{-1} \subset T$. It is easy to check that $g_{*} T^{*} g_{*}^{-1}$ also acts trivially on $G$. But as $g_{*} T^{*} g_{*}^{-1} \subset T \subset \operatorname{Aut}(G)$, the group $g_{*} T^{*} g_{*}^{-1}$, and so also $T^{*}$, has to be trivial.

This shows that $N=N^{*}$ is the maximal normal nilpotent analytic subgroup of $G^{*}$. Then, $\left[G^{*}, G^{*}\right] \subset N$, and $G^{*} / N$ is abelian and isomorphic to the direct sum $T \oplus(G / N)$. By the Theorem in $\S 5$ of Mostow $[\mathrm{M}], \pi \cdot N$ is closed in $G^{*}$. Therefore,

$$
\pi \cap N=\pi \cap N^{*}
$$

is a lattice in $N$.

Proof of Theorem A. We can now prove Theorem A, using the notations and the results of the lemmas above. that

As $G$ has the strong lattice property there is a lattice $\Lambda$ of $G$ with the property

$$
\Lambda \cap N=\pi^{*} \cap N=\Delta=\text { a lattice of the nilradical } N \text { of } G .
$$

Denote $A_{1}=\Lambda /(\Lambda \cap N) \subset G / N$ and $A_{2}=\pi^{*} /\left(\pi^{*} \cap N\right) \subset(G \rtimes T) / N$. There is an induced action of $(G \rtimes T) / N$ on each factor group $\gamma_{i}(N) / \gamma_{i+1}(N) \cong \mathbb{R}^{k_{i}}$ inducing a continuous representation $f_{i}:(G \rtimes T) / N \rightarrow \mathrm{GL}\left(k_{i}, \mathbb{R}\right)$.

Moreover, the representation $f_{i}$ is such that both $A_{1}$ and $A_{2}$ leave the lattice $\left(\Delta \cap \gamma_{i}(N)\right) /\left(\Delta \cap \gamma_{i+1}(N)\right)$ of $\gamma_{i}(N) / \gamma_{i+1}(N)$ fixed.

Now, the representation (where $c$ denotes the nilpotency class of $N$ )

$f=f_{1} \oplus f_{2} \oplus \cdots \oplus f_{c}:(G \rtimes T) / N \rightarrow \mathrm{GL}\left(k_{1}, \mathbb{R}\right) \oplus \cdots \oplus \mathrm{GL}\left(k_{c}, \mathbb{R}\right) \subset \mathrm{GL}\left(k_{1}+\cdots+k_{c}, \mathbb{R}\right)$

is a representation of $(G \rtimes T) / N$. We claim that this representation is faithful. We postpone however the proof of this claim until the end.

As $A_{1}$ and $A_{2}$ leave the same lattice of $\mathbb{R}^{k_{1}+\cdots+k_{c}}$ invariant, Lemma 2 implies that $G \cap \pi^{*}$ is of finite index in $\pi^{*}$, which finishes the proof, provided we can show that $f$ is a faithful representation of $(G \rtimes T) / N=G / N \oplus T$.

For any element $g \in G$, we will use $\bar{g}$ to denote its image in $G / N$. Now, let $g$ and $t$ be such that $f(\bar{g} t)=1$. As $\bar{g}$ and $t$ commute, we have that $f(\bar{g} t)=f(\bar{g}) f(t)=$ $f(t) f(\bar{g})$. The eigenvalues of $f(t)$ are all of modulus 1 and those of $f(\bar{g})$ are equal to 1 or of modulus different from 1 (property (3)(b) of groups of type (E) mentioned above). But as the eigenvalues of $f(\bar{g} t)$ (which are all supposed to be one) are obtained 
by multiplying the eigenvalues of $f(\bar{g})$ and $f(t)$ (as they commute), we must have that the eigenvalues of $f(\bar{g})$ are all equal to one. Now, Lemma 1 implies that $g \in N$ or that $\bar{g}=1$.

So, assume that $f(t)=1$. This means that the automorphism $t \in \operatorname{Aut}(G)$ restricts to a unipotent automorphism of $N$. As $t$ also acts trivially on $G / N$, the action of $t$ on $G$ is unipotent. However, the only element of $T$ acting unipotently on $G$ is the identity element, showing that $f$ is faithful.

THEOREM B. Let $G$ be a connected, simply connected solvable Lie group of type (E) with nilradical $N$, and let $G / N=\mathbb{R}^{n}$. Let $\rho: \mathbb{R}^{n} \rightarrow \operatorname{Out}(N)$ be the canonical representation. Assume:

The centralizer of $\rho\left(\mathbb{R}^{n}\right)$ in $\operatorname{Out}(N)$ has trivial maximal torus.

Then Statement 1 holds for this $G$ and hence $G$ has the strong lattice property.

Proof. In Lemma 3, $N=N^{*}$. Since $G^{*} / N$ is connected abelian, $G^{*} / N=\mathbb{R}^{n} \oplus T$, where $T$ is a torus. On the other hand, $G / N=\mathbb{R}^{n}$. Consider the natural representation $\rho^{*}: G^{*} / N=\mathbb{R}^{n} \oplus T \rightarrow \operatorname{Out}(N)$. This restricts to $\rho: G / N=\mathbb{R}^{n} \rightarrow \operatorname{Out}(N)$. Then $\rho\left(\mathbb{R}^{n}\right)$ commutes with $\rho^{*}(T)$. Since $\left.\rho^{*}\right|_{T}$ is injective, $T$ must be trivial by the hypothesis. This shows that $G^{*}=G$. By Lemma $3(1), \Gamma=\pi^{*}=\pi \cap G$ has finite index in $\pi$. By Remark 1, $G$ has the strong lattice property.

REMARK 2. Suppose there is a counter-example to the Statement 1 . Then one can find a subgroup $\pi^{*}$ of $\pi$, of finite index, such that the image of $\pi^{*}$ in $C$ has positive dimensional closure which is a torus. This implies that the centralizer of $\rho\left(\mathbb{R}^{n}\right)$ in $\operatorname{Out}(N)$ has non-trivial maximal torus, violating the condition of Theorem B.

3. Some examples of "bad" Lie groups. Below' we will list two examples of simply connected, connected solvable Lie groups $G$, for which the generalization of the first Bieberbach theorem (Theorem A) does not hold. The first example is easy, but consists of a Lie group which is not of type (E), while the second, slightly more sophisticated example consists of a Lie group of type $(\mathrm{R})$.

Example 1. There is an example in [A1], pp. 589-590, of dimension 5, where the first Bieberbach theorem does not hold. Here is a 3-dimensional example. Let $G=\mathbb{R}^{2} \rtimes \mathbb{R}$ be the universal covering group of $E_{0}(2)=\mathbb{R}^{2} \rtimes \mathrm{SO}(2)$. Therefore $t \in \mathbb{R}$ acts on $\mathbb{R}^{2}$ by

$$
\psi_{t}=\left(\begin{array}{rr}
\cos 2 \pi t & \sin 2 \pi t \\
-\sin 2 \pi t & \cos 2 \pi t
\end{array}\right)
$$

This solvable Lie group $G$ is not of type (E). Let $C=\mathrm{SO}(2)$, act on $\mathbb{R}^{2}$ in the standard way; that is, $e^{2 \pi i t} \in C$ acts via the matrix $\psi_{t}$. These two actions of $\mathbb{R}$ and $C$ on $\mathbb{R}^{2}$ commute with each other, so, there is an action of $C$ on $G$. Let $t_{0} \in \mathbb{R}$ be an irrational. Clearly, the element

$$
\gamma=\left(\psi_{t_{0}}, e^{-2 \pi i t_{0}}\right) \in G \rtimes C
$$

acts on $\mathbb{R}^{2}$ trivially. The $\psi_{t_{0}}$ part acts as the element of $G$, and the $e^{-2 \pi i t_{0}}$ part from $C$ undoes it. Take a lattice $\mathbb{Z}^{2}=\left\langle z_{1}, z_{2}\right\rangle$ of $\mathbb{R}^{2}$ and let it act as left translations. Consider the subgroup generated by $\mathbb{Z}^{2}$ and $\gamma$ :

$$
\pi=\left\langle z_{1}, z_{2}, \gamma\right\rangle \subset G \rtimes C .
$$

Then $\pi$ is isomorphic to $\mathbb{Z}^{3}$ and acts properly discontinuously on our $G$. It is obvious that $\pi$ does not contain any lattice of $G$ of finite index. Note that the image of $\pi$ in $C$ is dense. 
In this example $N^{*}=\mathbb{R}^{3}$, namely, it consists of all elements of the form $\left((x, y, z), e^{-2 \pi i z}\right)$, with $x, y, z \in \mathbb{R}$, while $N=\mathbb{R}^{2}$. It follows that $N^{*} / N \cong \mathbb{R}$. This shows that Lemma 3 is not valid for solvable Lie groups not being of type (E).

Look at the representation $\mathbb{R} \rightarrow \operatorname{Aut}\left(\mathbb{R}^{2}\right)=\mathrm{GL}(2, \mathbb{R})$. It has image $\mathrm{SO}(2)$, and its centralizer contains $\mathrm{SO}(2)$ itself. Thus the requirement in Theorem $\mathrm{B}$ is trivially not satisfied.

Example 2. Now, we give an example of a Lie group of type (R) for which the first Bieberbach theorem is not valid.

Consider the following matrix in $\operatorname{GL}(4, \mathbb{Z})$ :

$$
A=\left(\begin{array}{cccc}
0 & 1 & 0 & 0 \\
0 & 0 & 1 & 0 \\
0 & 0 & 0 & 1 \\
-1 & 1 & 1 & 1
\end{array}\right)
$$

This matrix has as characteristic polynomial $x^{4}-x^{3}-x^{2}-x+1$, having two real roots

$$
r_{1}, r_{2}=\frac{1}{4}(1+\sqrt{13} \pm \sqrt{2 \sqrt{13}-2})
$$

and two complex roots

$$
r_{3}, r_{4}=\frac{1}{4}(1-\sqrt{13} \pm i \sqrt{2 \sqrt{13}+2})
$$

for which following hold:

(1) $r_{1} r_{2}=1$, and $r_{3}=\overline{r_{4}}$, so $r_{3}$ is of modulus 1 .

(2) $r_{3}$ is not a root of unity.

There is a matrix $P \in \mathrm{GL}(4, \mathbb{R})$ such that

$$
\text { P.A. } P^{-1}=\left(\begin{array}{cccc}
r_{1} & 0 & 0 & 0 \\
0 & r_{2} & 0 & 0 \\
0 & 0 & b_{1} & b_{2} \\
0 & 0 & b_{3} & b_{4}
\end{array}\right)=\left(\begin{array}{cccc}
r_{1} & 0 & 0 & 0 \\
0 & r_{2} & 0 & 0 \\
0 & 0 & 1 & 0 \\
0 & 0 & 0 & 1
\end{array}\right)\left(\begin{array}{cccc}
1 & 0 & 0 & 0 \\
0 & 1 & 0 & 0 \\
0 & 0 & b_{1} & b_{2} \\
0 & 0 & b_{3} & b_{4}
\end{array}\right)=M_{1} M_{2}
$$

where the eigenvalues of $\left(\begin{array}{ll}b_{1} & b_{2} \\ b_{3} & b_{4}\end{array}\right)$ are $r_{3}$ and $r_{4}$. Note that $M_{1}$ and $M_{2}$ commute. We can define both $M_{1}^{t}$ and $M_{2}^{t}$ for a real parameter $t$. We remark that $M_{2}^{t+2 k \pi}=M_{2}^{t}$. Define

$$
\varphi: \mathbb{R} \rightarrow \mathrm{GL}(4, \mathbb{R}): t \mapsto P^{-1} M_{1}^{t} P
$$

and

$$
\psi: S^{1}=\mathbb{R} /(2 \pi \mathbb{Z}) \rightarrow \mathrm{GL}(4, \mathbb{R}): t \mapsto P^{-1} M_{2}^{t} P .
$$

It is obvious that $G=\mathbb{R}^{4} \rtimes \mathbb{R}$, where $\mathbb{R}$ acts on $\mathbb{R}^{4}$, via $\varphi$, is a solvable Lie group of type (R). Let $S^{1}=\mathbb{R} /(2 \pi \mathbb{Z})$ be the compact group of automorphisms of $G$ which is defined by taking $t(x, y)=(\psi(t)(x), y), \forall t \in \mathbb{R} /(2 \pi \mathbb{Z}), \forall x \in \mathbb{R}^{4}, \forall y \in \mathbb{R}$.

Now, the group $\Gamma=\mathbb{Z}^{4} \rtimes \mathbb{Z}$, where $1 \in \mathbb{Z}$ acts on $\mathbb{Z}^{4}$ as $A$, can be embedded into $G \rtimes S^{1}$, by mapping $\left(z_{1}, z_{2}\right) \mapsto\left(\left(z_{1}, z_{2}\right), z_{2}\right), \forall z_{1} \in \mathbb{Z}^{4}$ and $z_{2} \in \mathbb{Z}$. In this way $\Gamma$ is realized as a lattice of $G \rtimes S^{1}$ without $\Gamma \cap G=\mathbb{Z}^{4}$ being a lattice of $G$.

Look at the representation $\mathbb{R} \rightarrow \operatorname{Aut}\left(\mathbb{R}^{4}\right)=\mathrm{GL}(4, \mathbb{R})$. It has image $\mathbb{R}$ generated by $M_{1}$, and its centralizer contains $\mathrm{SO}(2)$ rotating the remaining 2-dimensional factors of $\mathbb{R}^{4}$. Thus the requirement in Theorem B is not satisfied. 
4. A class of "nice" Lie groups. Although Theorem A and B give us a necessary and sufficient condition for a Lie group of type (R) to admit a Bieberbach theory, they do not provide concrete criteria to check whether or not a given Lie group allows a generalization of the first Bieberbach theorem. Therefore, we describe in this section a substantial class of Lie groups of type $(R)$, satisfying the conditions of Theorem A.

DEFINITION 2. Let $G$ be a connected, simply connected solvable Lie group of type (R), with nilradical $N$. Then $G$ is said to be strongly filtered if and only if there exists a filtration of closed analytic, characteristic subgroups $N_{i}$ of $N$ :

$$
1=N_{0} \subseteq N_{1} \subseteq N_{2} \subseteq N_{3} \subseteq \ldots \subseteq N_{c}=N
$$

with $N_{i} / N_{i-1} \cong \mathbb{R}^{k_{i}}$, for all $i \in\{1,2, \ldots, c\}$ and such that

(1) If $\Gamma$ is a lattice of $N$ then $\left(\Gamma \cap N_{i}\right) /\left(\Gamma \cap N_{i-1}\right)$ is a lattice in $N_{i} / N_{i-1}$ for all $i \in\{1,2, \ldots, c\}$.

(2) the matrix representing the action of any element of $G / N$ on a factor $N_{i} / N_{i-1}$ is unipotent or has only positive real eigenvalues, each of multiplicity one.

REMARK 3. If $N$ is a connected and simply connected nilpotent Lie group, then both the upper central series and the lower central series of $N$ satisfy property (1) of the definition above. As property (2) is trivially satisfied in this case, $N$ is strongly filtered.

Example 3. Let $H$ be the three dimensional Heisenberg group consisting of matrices of the form $\left(\begin{array}{lll}1 & x & z \\ 0 & 1 & y \\ 0 & 0 & 1\end{array}\right)$, with $x, y, z \in \mathbb{R}$. There is an automorphism $\varphi_{1}$ of $H$ which is given by:

$$
\varphi_{1}\left(\begin{array}{lll}
1 & 1 & 0 \\
0 & 1 & 0 \\
0 & 0 & 1
\end{array}\right)=\left(\begin{array}{lll}
1 & 2 & 0 \\
0 & 1 & 1 \\
0 & 0 & 1
\end{array}\right), \varphi_{1}\left(\begin{array}{lll}
1 & 0 & 0 \\
0 & 1 & 1 \\
0 & 0 & 1
\end{array}\right)=\left(\begin{array}{lll}
1 & 1 & 0 \\
0 & 1 & 1 \\
0 & 0 & 1
\end{array}\right) \text { and } \varphi_{1}\left(\begin{array}{lll}
1 & 0 & 1 \\
0 & 1 & 0 \\
0 & 0 & 1
\end{array}\right)=\left(\begin{array}{lll}
1 & 0 & 1 \\
0 & 1 & 0 \\
0 & 0 & 1
\end{array}\right)
$$

This automorphism induces a commutative diagram

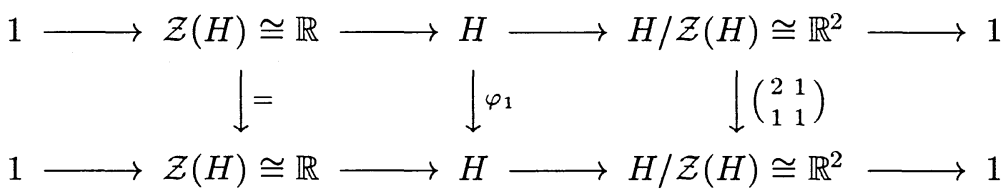

It follows that there is a morphism $\varphi: \mathbb{R} \rightarrow \operatorname{Aut}(H)$, with $\varphi(1)=\varphi_{1}$. Moreover, the resulting group $H \rtimes_{\varphi} \mathbb{R}$ is a lie group of type (R). Now, we define for all positive integers $k$, the following Lie group of type (R): $G_{k}=\mathbb{R}^{k} \times\left(H \rtimes_{\varphi} \mathbb{R}\right)$.

As both the upper and the lower central series of the nilradical of a Lie group of type $(\mathrm{R})$ satisfy the first criterion of the definition of a strongly filtered group, we focus on these filtrations of the nilradical $N=\mathbb{R}^{k} \times H$ of $G_{k}$.

The lower central series of $N$ is $1 \subseteq \mathbb{R}(=[N, N]=[H, H]) \subseteq N$, with $N / \mathbb{R} \cong \mathbb{R}^{k+2}$. Note that the action of an element of $G_{k} / N(\cong \mathbb{R})$ on $\mathbb{R}^{k+2}$ is not unipotent and has an eigenvalue 1 which is of multiplicity $k$. So, if $k>1$, this filtration of $N$, does not satisfy the second property of the definition of a strongly filtered group.

The upper central series of $N$ is $1 \subseteq \mathbb{R}^{k+1}\left(=\mathcal{Z}(N)=\mathbb{R}^{k} \times \mathcal{Z}(H)\right) \subseteq N$, with $N / \mathbb{R}^{k+1} \cong \mathbb{R}^{2}$. Now, the action of $G_{k} / N$ on $\mathbb{R}^{k+1}$ is unipotent, while each non-identity element of $G / N$ acts on $\mathbb{R}^{2}$ via an automorphism having two different eigenvalues. This implies that the upper central series of $G_{k}$ satisfies the second 
criterion of the definition of a strongly filtered group, which allows us to conclude that all groups $G_{k}$ are strongly filtered.

THEOREM C. Let $G$ be strongly filtered, then $G$ has the strong lattice property.

Before we are able to prove this theorem, we need to establish one more lemma

LEMmA 4. Let $\mathbb{Z}^{n} \subset \mathbb{R}^{n} \oplus T$, where $T$ is a torus, be a lattice such that the image $\mathbb{Z}^{n} \subset \mathbb{R}^{n} \oplus T \rightarrow T$ is dense in $T$. Let $f: \mathbb{R}^{n} \oplus T \rightarrow \mathrm{GL}(k, \mathbb{R})$ be a representation such that $f(x, 1)$ is unipotent or has only positive real eigenvalues, all being of multiplicity one, for all $x \in \mathbb{R}^{n}$. If $\mathbb{Z}^{n}$ leaves a lattice of $\mathbb{R}^{k}$ invariant, then the $T$ action on $\mathbb{R}^{k}$ is trivial.

Proof. The elements $f(1, t)(t \in T)$ all have eigenvalues which are of modulus one. As $T$ commutes with $\mathbb{R}^{n}$ it is known that the eigenvalues of $f(x, t)$ can be obtained as the product of the eigenvalues of $f(x, 1)$ and $f(1, t)$ (in some order).

Now, consider any element $\gamma=\left(x_{\gamma}, t_{\gamma}\right) \in \mathbb{Z}^{n}$. We claim that $f\left(1, t_{\gamma}\right)$ has only eigenvalues which are roots of unity. Indeed, suppose first that the action of $f(x, 1)$ only has positive real eigenvalues of multiplicity one. If $z$ is a complex eigenvalue of $f\left(x_{\gamma}, t_{\gamma}\right)$, then also $\bar{z}$, the complex conjugate of $z$, is an eigenvalue of $f\left(x_{\gamma}, t_{\gamma}\right)$. However, as the eigenvalues of $f\left(x_{\gamma}, t_{\gamma}\right)$ are obtained by multiplying the eigenvalues of $f\left(x_{\gamma}, 1\right)$ with the eigenvalues of $f\left(1, t_{\gamma}\right)$, all these eigenvalues have a different modulus. This implies that $z=\bar{z} \in \mathbb{R}$. But this implies in turn that $f\left(1, t_{\gamma}\right)$ has only 1 or -1 as eigenvalues.

Now, suppose that the action of $f\left(x_{\gamma}, 1\right)$ is unipotent, then all eigenvalues of $f\left(x_{\gamma}, t_{\gamma}\right)$ are of modulus 1 . As $f\left(x_{\gamma}, t_{\gamma}\right)$ fixes a lattice of $\mathbb{R}^{k}$, the matrix $f\left(x_{\gamma}, t_{\gamma}\right)$ is conjugated, inside $\operatorname{GL}(k, \mathbb{R})$, to a matrix in $\operatorname{GL}(k, \mathbb{Z})$, which has of course the same eigenvalues. Now [ST]; Lemma 11.6 implies that all eigenvalues of $f\left(x_{\gamma}, t_{\gamma}\right)$, which are the same as the eigenvalues of $f\left(1, t_{\gamma}\right)$ are roots of unity.

As $\mathbb{Z}^{n}$ is finitely generated, there is a subgroup of finite index in $\mathbb{Z}^{n}$, for which the elements $\left(x_{\gamma}, t_{\gamma}\right)$ are such that the eigenvalues of $f\left(1, t_{\gamma}\right)$ are all 1 . As this set of $t_{\gamma}$ 's is still dense in $T$, it follows that the eigenvalues of $f(1, t)$ for all elements of $t \in T$ must be 1 . This means that $T$ acts unipotently on $\mathbb{R}^{k}$. However, the only unipotent action of $T$ on $\mathbb{R}^{k}$ is the trivial action, which finishes the proof of the lemma.

Proof of Theorem $C$. Let $T$ be a torus in $\operatorname{Aut}(G)$ and choose a lattice $\pi$ in $G \rtimes T$. As before, we let $G^{*}$ be the identity component of the closure of $\pi G$ in $G \rtimes T$ and we let $\pi^{*}=G^{*} \cap \pi$. We know that $G^{*}=G \rtimes T^{\prime}$ for some torus $T^{\prime} \subset \operatorname{Aut}(G)$ and by Lemma 3 , we have that

(1) $\pi^{*}$ is of finite index in $\pi$,

(2) the nilradical of $G^{*}$ coincides with the nilradical $N$ of $G$ and

(3) $\pi^{*} \cap N=\pi \cap N$ is a lattice of $N$.

We will show that $T^{\prime}$ is the trivial group. Suppose that

$$
1=N_{0} \subseteq N_{1} \subseteq N_{2} \subseteq N_{3} \subseteq \ldots \subseteq N_{c}=N
$$

is the filtration satisfying the properties of the definition of a strongly filtered Lie group of type (R). As $\pi^{*} /\left(\pi^{*} \cap N\right)$ is a lattice of $G / N \times T^{\prime}$, it follows from Lemma 4 that $T^{\prime}$ acts trivially on any quotient $N_{i} / N_{i-1}(1 \leq i \leq c)$. Therefore, $T^{\prime}$ acts unipotently on $N$. However, as said before, this implies that the action of $T^{\prime}$ on $N$ is trivial. This combined with the fact that $T^{\prime}$ acts trivially on $G / N=\mathbb{R}^{n}$ implies that $T^{\prime}$ acts trivially on $G$. But as $T^{\prime} \subseteq \operatorname{Aut}(G)$, this implies that $T^{\prime}$ is trivial.

Hence $G=G^{*}$ and $\pi^{*}=\Gamma=\pi \cap G$ itself is a lattice of $G$, with $\Gamma \cap N=\pi \cap N$.

This completes the proof of the theorem. 
REMARK 4. If $G$ is a nilpotent connected and simply connected Lie group, the conditions of the above theorem are automatically satisfied.

\section{REFERENCES}

[A1] L. AUSLANDER, Bieberbach's theorems on space groups and discrete uniform subgroups of Lie groups, Annals of Math., 71 (1960), pp. 579-590.

[A2] L. AUSLANDER, Bieberbach's theorems on space groups and discrete uniform subgroups of Lie groups II, American J. Math., 83 (1961), pp. 276-280.

[C] Leonard S. Charlap, Bieberbach Groups and Flat Manifolds, Springer-Verlag, New York Inc. (Universitext), 1986.

[D1] Karel Dekimpe, Determining the translational part of the fundamental group of an infrasolvmanifold of type (R)., Math. Proc. Cambridge Ph. Soc., 122, pp. 515-524.

[D2] Karel Dekimpe, Almost-Bieberbach Groups: Affine and Polynomial Structures, Lecture Notes in Mathematics 1639, Springer-Verlag, Heidelberg, 1996.

[DIM] Karel Dekimpe, Paul Igodt, and Wim Malfait, There are only finitely many infranilmanifolds under each nilmanifold: a new proof., Indag. Math. (N.S.), 5 (1994), pp. 259-266.

[G1] V. V. GoRBACEvič, Discrete subgroups of solvable Lie groups of type (E), Math. USSR Sbornik, 14:2 (1971), pp. 233-251.

[G2] V. V. GoRBACEVIČ, Lattices in solvable Lie groups and deformations of homogeneous spaces, Math. USSR Sbornik, 20:2 (1973), pp. 249-266.

[KT] F. W. Kamber and Ph. Tondeur, Flat manifolds with parallel torsion, J. Differential Geometry, 2 (1968), pp. 385-389.

[L] K. B. LEE, Infra-solvmanifolds of type (R), Quarterly J. Math., Oxford(2), 46 (1995), pp. 185195.

[LR1] K. B. Lee And F. Raymond, Seifert manifolds with $\Gamma \backslash G / K$-fiber, Michigan Math. Journal, 43:3 (1996), pp. 437-464.

[LR2] K. B. LeE AND F. RAYmond, Geometric realization of group extensions by the Seifert construction, Contemporary Math. A.M.S., 44 (1985), pp. 353-411.

[M] G. D. Mostow, Factor spaces of solvable groups, Annals of Math., 60 (1954), pp. 1-27.

[OV] A. L. OnishchiK And E. B. Vinberg (EDs.), Lie Groups and Lie Algebras III, Encyclopedia of Mathematical Sciences 41, Springer-Verlag, Berlin, 1994.

[ST] I. N. Stewart and D. O. Tall, Algebraic Number Theory, 2nd Edition, Chapman and Hall, New York, 1987.

[W] J. Wolf, Spaces of Constant Curvature, 4th Edition, Publish or Perish, 1977. 
